\title{
Cochlear Implant Considerations in Children with Additional Disabilities
}

\author{
C. Eduardo Corrales $\cdot$ John S. Oghalai
}

Published online: 1 February 2013

(c) Springer Science+Business Media New York 2013

\begin{abstract}
Early identification and management of disabilities in children are essential to reduce long-term developmental sequelae. Many of the causes of hearing loss also produce cognitive delays resulting in a large number of children with both deafness and developmental disabilities. Children who have hearing loss and additional disabilities require complex, individualized therapy to maximize their long-term quality of life. Hearing loss is often detected early because of widespread newborn hearing screening programs and the decision for cochlear implantation in children presenting with multiple medical and developmental disorders is still evolving. This article will review the literature regarding cochlear implant considerations in children with additional developmental disabilities in areas of family perception, speech and language development, cognitive development including adaptive behavior and intelligence, communication and functional skills, auditory outcomes, quality of life outcomes, predictors of outcomes and realistic expectations after cochlear implantation.
\end{abstract}

Keywords Cochlear implant - Developmental delay . Disability · Hearing loss · Otorhinolaryngology ·

Children · Pediatric

C. E. Corrales · J. S. Oghalai $(\square)$

Department of Otolaryngology-Head and Neck Surgery,

Stanford University School of Medicine, 801 Welch Road,

Stanford, CA 94305, USA

e-mail: joghalai@stanford.edu

C. E. Corrales

e-mail: educor@stanford.edu

\section{Introduction}

Children with hearing loss and additional developmental disabilities challenge the cochlear implant team's ability to predict the likely benefit of a cochlear implant. As cochlear implant centers expand their implant criteria and become more comfortable implanting children with additional disabilities, assessment tools and outcomes are being identified to better understand and support this specific population. Understanding the specific needs of this population and family perceptions of benefit is essential to providing the best services and opportunities for a child's success with a cochlear implant.

Hearing loss is the fourth most common developmental disorder in the United States and deafness is the most common sensory disorder [1]. Approximately $2-3$ in 1,000 children are born with a significant degree of sensorineural hearing loss [2-4]. The prevalence of congenital hearing loss is greater than twice that of all other diseases and syndromes routinely screened at birth combined [5]. Hence, universal newborn hearing screening programs have been widely implemented [2]. It is estimated that about $25 \%$ of the cases of congenital hearing loss are attributed to identifiable prenatal or postnatal disease or trauma, $18 \%$ to undiagnosed genetic factors, $15 \%$ to autosomal dominant genetic mutations, $40 \%$ to autosomal recessive genetic mutations and $2 \%$ to sex-linked genetic mutations [6]. Prematurity and very low birth weight are also risk factors for sensorineural hearing loss [7, 8]. The motivations for knowing the etiology of hearing loss is important for numerous purposes: prediction of worsening hearing loss over time, to determine if the level of hearing loss might improve with the use of a hearing aid or cochlear implant, and if a genetic cause is found, HL could be a part of a syndrome and also help prepare for genetic counseling $[9,10]$. 
This article will focus on recent data on key domains used to measure cochlear implant outcomes in children with multiple disabilities, and thus provide a framework in which implant teams can provide families with appropriate expectations and guidance.

\section{Cognition in Children with Sensorineural Hearing Loss}

The ability to hear during the early years of life is critical for the development of speech, language and cognition. Early identification and intervention can prevent severe psychosocial, educational, and linguistic repercussions [11, 12]. Much research has been done on cognitive abilities and behavior adaptability in hearing children. Research has demonstrated that intelligence and adaptive behaviors are highly related, meaning that adaptive behavior can be predictive of certain aspects of future performance such as socioeconomic functioning and integration into the community [13]. This body of research has also been applied to children with profound sensorineural hearing loss. Results from multiple studies suggest that early identification of the child's deafness and initiation of early intervention services predicted better language development. Better language development leads to better parental communication that improves outcome measures in behavior adaptability and cognitive skills. For example, deaf children who are not enrolled in early sign language or any other visual communication or non-verbal form of communication (eye contact or visual cues) demonstrate deprived self-regulation (unable to appropriately internalize social cues) and emotional development [14]. With reduced self-regulation and absent communication feedback, deaf preschoolers are not able to express their necessities and ultimately externalize their emotions and thoughts through altered behaviors [14]. There is evidence of decreased gross motor skills in children with sensorineural hearing loss as compared with normal-hearing peers where deaf children were found to have decreased balance and complex motor movements [15]. The impact of auditory deprivation in toddlers and preschoolers also causes a significant decline in visual reception skills and fine motor skills with advancing age [10]. Additionally, nonverbal reasoning, planning, and visual awareness are decreased in prelingually deafened children who are free of neurological impairment compared to same-age normal hearing children [15].

\section{Developmental Delay (DD) and Prematurity}

More than $30 \%$ of children who present with sensorineural hearing loss have additional developmental disabilities $[16 \bullet \cdot, 17,18]$. Developmental delay (DD) is a pattern of persistently slow learning of basic motor and language skills during childhood and includes diminished intelligence and adaptive abilities (the ability to function and cope within the environment) $\left[19,20^{\bullet}\right]$. Disabilities may include DD, visual and visual/spatial disorder, speech and language impairment, physical and gross motor disorders including cerebral palsy, autism and attention deficit hyperactive disorder. Common causes of DD include syndromes (CHARGE, Down, Fragile X), hypoxia at birth, CNS infections (CMV [21], meningitis), problems during pregnancy (fetal alcohol syndrome and rubella infection), neurological syndromes (cerebral palsy [22], epilepsy), malnutrition, and brain trauma during birth. The two most common etiologies for DD in cochlear implant patients are CHARGE syndrome and CMV infection. Additionally, contributing to the problem is the fact that certain developmental disabilities are not evident early in life [17].

The survival rate of premature infants increased dramatically during the latter half of the twentieth century, although recently it has leveled off [23]. There has been an increasing awareness of the importance of identifying and managing the long-term sequelae found in the survivors. Premature infants are at a particularly high risk of multiple handicaps which affect roughly $36 \%$ of children born at $<26$ weeks gestational age [24]. Other high-risk populations of having developmental disabilities include very low birth weight and oxygen dependency at 36 weeks [7, $20 \bullet, 25]$. Hence, DD is the most common developmental disorder in the United States [1].

\section{Cochlear Implantation in Children with Additional Disabilities}

Severe to profoundly deaf children with normal cognition have routinely been implanted since 1980 [26]. However, cochlear implants (CI) have typically only been placed sporadically in children with additional disabilities. This is because of the traditional clinical aim of auditory habilitation of patients with congenital hearing loss is to achieve oral communication. However, children with multiple handicaps have a decreased ability to learn speech and language even with normal hearing. For a child with both hearing loss and multiple disabilities, DD is the ultimate limiting disability affecting the child's outcome. Thus, many centers do not routinely offer CI to children that are expected to have substantial difficulty in developing speech and language. As cochlear implant centers have expanded their selection criteria for implantation and have become more confident in pediatric cochlear implantation, the numbers of CI performed in deaf children with additional disabilities has increased. The few reports describing outcomes in this patient population suggest that cochlear 
implantation produces increases in speech intelligibility, acquisition of language skills, development of adaptive behavior, and possibly cognitive development [20•, 27•].

Additional disabilities, such as hearing and cognitive delay, interact synergistically to magnify the adverse effect on development. Such a phenomenon has been termed a pseudo-handicap effect where more than one disability greatly increases the overall disability more than might be expected $[10,17]$.

\section{Family Perception in Children with Additional Disabilities after Cochlear Implantation}

Families of children with additional disabilities perceive significant benefits attributed to their child's cochlear implant as studied by Wiley et al. [18]. Perceived benefits in this study included awareness to environment sounds (94\% of the children), more likely to communicate wants/ needs $(88 \%)$, developing speaking skills $(88 \%)$, more attentive and interested at home (82\%), getting along better with classmates $(74 \%)$ and siblings $(71 \%)$.

Similar results were obtained in a more recent study [28] using the same questionnaire used by Wiley et al. [18]. This study group consisted of 23 children with additional disabilities before and after implantation, with 10 out of the 23 patients having mental retardation. All parents reported an improved awareness to environmental sounds, $74 \%$ indicated improvements in the child's speaking skills and $96 \%$ reported improved interaction with peers [28]. Additionally, $96 \%$ of families reported that their child was more likely to communicate their want or needs, $100 \%$ reported the child to be more attentive/interested at home and in school and $100 \%$ reported working better with siblings and classmates. When only the families of children with mental retardation were asked for perceived benefits after cochlear implant, $100 \%$ responded increase awareness of environmental sounds, $70 \%$ improved development of speaking skills, $100 \%$ improvement in peer interactions, $100 \%$ indicated that their child was more likely to communicate their needs or wants, more attentive and interested at home and school, and worked better with siblings and classmates [28].

Palmieri et al. [29•] have recently advanced the Deafness and Additional Disabilities questionnaire (DADQ) as a tool developed based on the concept that an improvement in the quality of life of deaf children with additional disabilities with CI derives from increased well being in the following domains: physical, neuropsychological and social. The questionnaire consists of five areas: perceptual skills (perception and identification of environmental sounds, speech perception in quiet and noisy environments), preferred mode of communication (scored from 0 [behavior] to 8 [use of full sentences]), communicative behaviors (child's communicative behaviors with familiar talkers and strangers in relation to the child's age and language development), attention and memory (focused and selective attention to long-term memory skills) and social interaction, control of behavior and self-government (child's behavior during interaction with family and friends and with siblings and peers, emotional needs, independence in daily activities). The DADQ was administered before and after cochlear implantation in 50 children with additional disabilities. Their results show that children with additional disabilities have substantial progress in all areas of the DADQ. The largest improvements were seen in the perception skills, followed by preferred mode of communication, followed by communicative behaviors and lastly, attention and memory skills. The domains of social interaction, control of behavior and selfgovernment improved significantly less.

\section{Outcome Measures}

Cognitive Development after Cochlear Implantation in Children with Additional Disabilities

The main theme arising from studies investigating cognitive outcome measures in implanted children with additional disabilities is that the greater the DD, the poorer the outcome measures. This has been studied in detail. Using the standardized Mullen Scales of Early Learning (MSEL), which is a test to estimate intelligence, children with DD showed less comprehensive developmental improvements after implantation [20•]. Two factors were attributed to this result: (1) children with DD were implanted later, likely due to other comorbidities delaying their implantation and (2) they initially start off with a lower level of intelligence. In a similar manner, using the Vineland Adaptive Behavior Scale (VABS), which measures adaptive behavior in children with additional disabilities started off with lower scores but after cochlear implantation, these patients did not lag further behind during the follow up period. This suggests that a normal developmental acquisition rate of adaptive behavior was seen [20•].

Auditory Skills after Cochlear Implantation in Children with Additional Disabilities

The auditory skills checklist (ASC) is a criteria-referenced assessment tool that uses a combination of a preformatted parent interview and clinical observation to determine functional auditory skill development $[30,31]$. It has been validated in children with $\mathrm{CI}$, and it has been applied to implanted children with additional disabilities. Results of this study demonstrated that children with additional 
disabilities made progress in auditory skills, regardless of disability type [31]. But this measurable progress was significantly modified by the degree of DD.

Categories of auditory performance (CAP) is a scale used to rate outcomes from post-implanted children. It provides a scale on which children's developing auditory abilities can be rated in eight categories in order of increasing frequency. Scores $0-4$ have varying degrees of identifying sounds but not verbal language spoken by the patient. Scores between 5 and 7 achieve varying complexities of verbal language use. This scale has also been applied to post-implanted children with additional disabilities [17]. At 12 months post implantation, $96 \%$ children without disabilities had a CAP score of 5 or greater in speech compared with $52 \%$ of children with additional disabilities. The median CAP score at 12 months of children with DD was 4 compared to 6 in children without DD. Again, additional disabilities significantly affect the outcomes of CI with "slower progress, less predictable outcomes and poorer results" [17]. However, cochlear implantation in children with additional disabilities provides several benefits including environmental sound awareness and recognizing the parent's voice, greater social interactions and for some language understanding and speech $[10,17,27 \cdot]$.

Speech Perception and Language Development after Cochlear in Children with Additional Disabilities

As expressed earlier, language acquisition may not be the ultimate goal of an implant for children with additional disabilities. Speech and language acquisition is of high priority and the evidence suggests increased strides in both areas after cochlear implantation. Meinzen-Derr et al. [32•] reported a small group of post-implant patients with DD that improved on either expressive or receptive language skills over time. A high correlation between the non-verbal cognitive quotient (NVCQ; of the Revised Gesell Developmental Schedule) and expressive and receptive language quotients was established. These findings have important clinical implications: early measures of nonverbal cognition were highly predictive of language outcomes postimplantation. Wiley et al. [27•] also reported gains in language age skills in both receptive and expressive domains in post-implant children with DD. But when looking at the language trajectory, their language quotients did not increase, meaning that even though children made headway, these gains were not sufficient to keep up with their age.

The speech intelligibility rating (SIR) is a scale that has been adapted for use with post-implanted children and found to be reliable for measuring speech intelligibility. The E2L is a screening test of speech perception designed for clinical practice [33]. Edwards et al. [33] used these two outcome measures to study a group of children with additional disabilities at 1 and 2 years post-implantation. In all the statistical analyses performed, the best predictors of speech intelligibility and speech perception were variables related to the degree of DD. The greater the developmental disability, the poorer the speech intelligibility and speech perception outcomes [33]. Holt and Kirk [34] specifically measured speech and language outcomes in cognitively delayed children after cochlear implantation. Children with mild developmental delays do make measurable progress in both speech intelligibility and speech perception.

Predictors of spoken language development following cochlear implantation have been evaluated in a study at 1,2 and 3 years post implantation [35]. All children in this study had normal intelligence (cognitive impaired children were excluded), but one-fourth of children had additional disabilities including motor/balance disorder, behavioral problems (ADHD, autism), learning disorders (dyslexia), auditory neuropathy and multiple handicaps (a combination of additional disabilities). In terms of receptive language, children who had a learning disability had significantly lower scores at 1, 2 and 3 years post implantation. In regards to expressive language with word development, children with additional disabilities had lower scores than children without disabilities regardless of the etiology of the disability through 1,2 and 3 years after implantation. When testing expressive language with sentence development, children with a learning disability consistently scored lower than other disabilities and children with no disabilities.

The six speech perception categories developed by Geers and Moog [36] have been applied to 23 children with additional disabilities before and after cochlear implantation [28]. This scale consists of seven categories: 0-6 where 0 denotes no pattern of speech perception and 6 denotes open set recognition of words. Before implantation, most children fell in categories $0(48 \%)$ or 1 (26\%). After implantation only $13 \%$ remained in categories 1 or 2 and $53 \%$ attained speech perception abilities in category 6 . Additionally, a group of children with mental retardation were also assigned categories in the speech perception scale. Before implantation, $60 \%$ of children were given categories 0 and 1 , and $40 \%$ were given categories 2-5. After implantation, $50 \%$ of children achieved category 6 , and only $20 \%$ remained assigned to category 0 or 1 [28].

Nikolopoulos et al. [37••] used the SIR scale to evaluate implanted children with additional disabilities and compared them with age-equivalent implanted children without such disorders in a follow up period of 5 years. Their results showed that $70 \%$ of children with additional disabilities reached connected intelligible speech versus $96 \%$ 
in implanted children without additional disabilities. However, the quality of speech was significantly different between both groups. Only $11 \%$ of children with additional disabilities reached the highest categories. In comparison, $66 \%$ if implanted children without additional disabilities reached these higher categories. The strongest correlation with this outcome was the total number of additional disabilities, and in these, language and communication disorders were the most contributing factors. Their conclusion was that the majority of deaf children with additional disabilities develop connected intelligible speech 5 years after CI, but that a third did not develop any speech at all, thus the benefit of cochlear implantation should not be exclusively restricted to speech production alone.

Meinzen-Derr et al. [16••] evaluated the differences in language skills after cochlear implantation in children with additional disabilities compared to age-matched hearing children with similar disabilities. The results of their study showed that although implanted children with multiple disabilities increase their language skills, they underscore compared to developmentally matched hearing children. This finding has an important and direct clinical implication. Post-implanted children with additional disabilities had significantly lower language levels with delays disproportionate to their cognitive potential and warrants closer attention since these patients might require more frequent checkups of language and communication therapy, or changes in their therapy management.

\section{Communication Skills after Cochlear Implantation in Children with Additional Disabilities}

The APCEI-scale (Acceptation, Perception, Comprehension, oral Expression, speech Intelligibility) evaluates five components of language: cochlear implant acceptance, perceptive language performance, comprehension of the oral orders, expressive language and speech intelligibility. The corrected APCEI is used when comparing expected outcomes in different age groups. The APCEI scale has been used to determine prognostic factors of implanted children regarding communication skill progress [38]. Gerard et al. [38] studied a group of 89 implanted children using the APCEI scale. Half of the cohorts were children with additional disabilities. The presence of an associated disability was linked with a slower progress in the APCEI scores from implantation to 3 years after implantation compared with children without a disability. But, even if their progress is slower, children with additional disabilities derived substantial benefit and continued to improve their communication skills over time. In fact, in their study, they show an equivalent parallel trajectory of development compared to children without disabilities and that over time, the gap is further reduced.

Communication skills of children with additional disabilities have also been evaluated by asking the parents how their child communicates before and after the implant [18]. Communication skills measured were behavior only (crying, facial expressions, gestures), behavior and sign (behaviors and signs to communicate), sign only (uses formal sign system), sign and oral (combination of sign language and spoken words) and oral (child only communicates orally). The children made significant measurable progress in communication skills after cochlear implantation. All children increased their communication skills, and $50 \%$ were assigned a higher type of communication; though some remained within their pre-implant category, meaning that they broadened their skills but not enough to be placed into the next category. Although not all of the children with additional disabilities achieved oral and language abilities, all broadened their communication skills [18].

Palmieri et al. [29•] used the DAD questionnaire (described above) to assess the preferred way a child uses to communicate before and after cochlear implantation. The 'preferred mode of communication' section of the DADQ is scored from 0 to 8 , where 0 denotes the lowest score and represents smiles, cries and facial expressions to communicate, and 8 denotes children who use complete sentences for communication. Before implantation, $82 \%$ of the children used behavior, gestures or vocalization (score 0-2) as a preferred mode of communication and only $18 \%$ used oral language (score 8 ). After implantation, $80 \%$ of children used oral language of which $28 \%$ used association of words (score 7) and $32 \%$ used complete sentences (score of 8 ).

Berrettini et al. [28] used a similar categorization for modes of communication, but only had five categories instead of eight: 1-5 where 1 signifies behavior communication (cries, facial expressions) and 5 signifies exclusively use of oral language. Before implantation, the main mode of communication in $69 \%$ of patients was behaviors and gestures (score 1 and 2), and $28 \%$ used oral language (score 4 and 5). After implantation, $28 \%$ of children were using behaviors and gestures (score 1 and 2) and $69 \%$ were using oral language (score 4 and 5). They separated all patients with mental retardation, and assessed their main mode of communication before and after implantation. Before implantation, $70 \%$ of patients with mental retardation used behaviors and gestures (score 1 and 2) as a main mode of communication, and $30 \%$ used gestures and oral language (score 4). After implantation, $70 \%$ of children with mental retardation were using oral language (exclusively or associated to gestures-score 4 or 5) [28]. 
Functional Skills after Cochlear Implantation in Children with Additional Disabilities

The pediatric evaluation of disability inventory (PEDI), which is a standardized test for essential daily functional activities, contains measures of Self-Care, Mobility and Social Function. Wiley et al. [27•] showed measurable improvements in all three domains comparing pre-implant and 1-year post-implant children with DD. An important observation in this study was that even though children with DD had measurable progress, the median standard on the Self-Care and Social Function domains decreased over time, highlighting the importance of frequent functional assessments in children with DD. More importantly, this observation was also seen regarding language acquisition where children with DD had progressive increase in language measures but not at the rate relative to their age. Still, language abilities had an important impact on functioning in the domains of self-care and social function.

Quality of Life after Cochlear Implantation

in Children with Additional Disabilities

The overriding appeal of quality of life assessments are that they are consistent with the holistic concept of rehabilitation and the realization that certain limitations will always exist. The quality of life of a child is typically associated with the quality of life of the parents, and both have been successfully measured for children with cognitive delays [39]. The Parenting Stress Index (Psychological Assessment Resources, 4th ed) (PSI) is designed to objectively evaluate the magnitude of stress in the parent-child system. It focuses on three major domains of stress: child characteristic, parent characteristics and situational/demographic life stress. It can be used in children from 1 month to 12 years of age and requires that parents read and speak English. Within the parent domain, seven subscales (Competence, Isolation, Attachment, Health, Role Restriction, Depression, and Spouse/Parenting Partner Relationship) measure sources of stress related to parent characteristics. Within the child domain, six subscales (Distractibility/Hyperactivity, Adaptability, Reinforces Parent, Demandingness, Mood, and Acceptability) evaluate sources of stress as gathered from the parent's report of child characteristics. Recently, the PSI has been studied in implanted children with and without DD [20•]. Before cochlear implantation, children with and without DD had similar initial PSI scores, suggesting that there were similar levels of stress in families of both groups. After cochlear implantation, PSI scores showed that children with DD had increase stress in the child domain, whereas children without DD did not. This implies an increase in problematic behavior patterns affecting the parenting system in children with DD. However, in this study, the children with DD were implanted at an older age compared to children without DD (median 240 months vs 16 months, respectively). After statistical accounting for the later age of implantation, the stress level as measured by PSI resolved.

\section{Conclusion}

Predicting outcomes of $\mathrm{CI}$ in children with additional disabilities is an inherently difficult task. Parents who are considering cochlear implantation for their child with additional disabilities naturally want to know the degree of benefit their child will receive. Thus, parental expectations of a child with additional disabilities after cochlear implantation should be appropriate and realistic in terms of what the CI might provide. There is no clear agreement on the definition of benefit for children with additional disabilities who get implanted. Most authors agree that appropriate expectations should include improved sound awareness, increased personal and social interactions, more environmental connectivity, higher quality of life, and family satisfaction. The development of oral communication skills would not typically be an appropriate expectation after implantation in a child with developmental delays. However, every child has individual strengths and weaknesses that need to be assessed as part of the cochlear implant candidacy evaluation and counseling process.

In general, implanted children with additional disabilities consisting of mild cognitive and developmental delay, make significant progress comparable to their peers. However, children with disabilities consisting of significant learning impairment and severe global developmental delay fare less favorable, but improved sound awareness and better interaction with their milieu is a realistic and appropriate parental expectation.

Acknowledgments This work was supported by National Institutes of Health grant R01 DC010075.

Disclosure No potential conflicts of interest relevant to this article were reported.

\section{References}

Papers of particular interest, published recently, have been highlighted as:

- Of importance

•- Of major importance

1. Bhasin TK, Brocksen S, Avchen RN, Van Naarden Braun K. Prevalence of four developmental disabilities among children aged 8 years-Metropolitan Atlanta Developmental Disabilities Surveillance Program, 1996 and 2000. MMWR Surveill Summ. 2006;55(1):1-9. 
2. Morton CC, Nance WE. Newborn hearing screening-a silent revolution. N Engl J Med. 2006;354(20):2151-64.

3. Haggard MP, Pullan CR. Staffing and structure for paediatric audiology services in hospital and community units. Br J Audiol. 1989;23(2):99-116.

4. Yoon PJ. Pediatric cochlear implantation. Curr Opin Pediatr. 2011;23(3):346-50.

5. Finitzo T, Crumley WG. The role of the pediatrician in hearing loss. From detection to connection. Pediatr Clin North Am. 1999;46(1):15-34.

6. Steel KP, Brown SD. Genetics of deafness. Curr Opin Neurobiol. 1996;6(4):520-5.

7. Cristobal R, Oghalai JS. Hearing loss in children with very low birth weight: current review of epidemiology and pathophysiology. Arch Dis Child Fetal Neonatal Ed. 2008;93(6):F462-8.

8. Oghalai JS, Chen L, Brennan ML, Tonini R, Manolidis S. Neonatal hearing loss in the indigent. Laryngoscope. 2002;112(2): 281-6.

9. Jerry J, Oghalai JS. Towards an etiologic diagnosis: assessing the patient with hearing loss. Adv Otorhinolaryngol. 2011;70:28-36.

10. Pierson SK, Caudle SE, Krull KR, Haymond J, Tonini R, Oghalai JS. Cognition in children with sensorineural hearing loss: etiologic considerations. Laryngoscope. 2007;117(9):1661-5.

11. Moeller MP. Early intervention and language development in children who are deaf and hard of hearing. Pediatrics. 2000; 106(3):E43.

12. Bubbico L, Di Castelbianco FB, Tangucci M, Salvinelli F. Early hearing detection and intervention in children with prelingual deafness, effects on language development. Minerva Pediatr. 2007;59(4):307-13.

13. McIntyre LL, Blacher J, Baker BL. The transition to school: adaptation in young children with and without intellectual disability. J Intellect Disabil Res. 2006;50(Pt 5):349-61.

14. Kushalnagar P, Krull K, Hannay J, Mehta P, Caudle S, Oghalai J. Intelligence, parental depression, and behavior adaptability in deaf children being considered for cochlear implantation. J Deaf Stud Deaf Educ. 2007;12(3):335-49.

15. Schlumberger E, Narbona J, Manrique M. Non-verbal development of children with deafness with and without cochlear implants. Dev Med Child Neurol. 2004;46(9):599-606.

16. •• Meinzen-Derr J, Wiley S, Grether S, Choo DI. Children with cochlear implants and developmental disabilities: a language skills study with developmentally matched hearing peers. Res Dev disabil. 2011;32(2):757-767. Evaluated the differences in language skills after cochlear implantation in children with additional disabilities compared to age-matched hearing children with similar disabilities.

17. Birman CS, Elliott EJ, Gibson WP. Pediatric cochlear implants: additional disabilities prevalence, risk factors, and effect on language outcomes. OtolNeurotol. 2012;33(8):1347-52.

18. Wiley S, Jahnke M, Meinzen-Derr J, Choo D. Perceived qualitative benefits of cochlear implants in children with multi-handicaps. Int J Pediatr Otorhinolaryngol. 2005;69(6):791-8.

19. American Psychiatric Association. American Psychiatric Association. Task Force on DSM-IV. Diagnostic and statistical manual of mental disorders : DSM-IV-TR. 4th ed. Washington, DC: American Psychiatric Association; 2000.

20. - Oghalai JS, Caudle SE, Bentley B, et al. Cognitive outcomes and familial stress after cochlear implantation in deaf children with and without developmental delays. Otology Neurotol.: official publication of the American Otological Society, American Neurotology Society [and] European Academy of Otology and Neurotology. 2012;33(6):947-956. Uses the MSEL and VABS tests to compare cognitive outcomes in implanted children with additional disabilities.
21. Yamazaki H, Yamamoto R, Moroto S, et al. Cochlear implantation in children with congenital cytomegalovirus infection accompanied by psycho-neurological disorders. Acta Otolaryngol. 2012;132(4):420-7.

22. Bacciu A, Pasanisi E, Vincenti V, et al. Cochlear implantation in children with cerebral palsy. A preliminary report. Int J Pediatr Otorhinolaryngol. 2009;73(5):717-21.

23. Fanaroff AA, Stoll BJ, Wright LL, et al. Trends in neonatal morbidity and mortality for very low birthweight infants. Am J Obstet Gynecol. 2007;196(2):147.

24. Xiong T, Gonzalez F, Mu DZ. An overview of risk factors for poor neurodevelopmental outcome associated with prematurity. World J Pediatr. 2012;8(4):293-300.

25. Taylor HG, Klein N, Drotar D, Schluchter M, Hack M. Consequences and risks of $<1000$-g birth weight for neuropsychological skills, achievement, and adaptive functioning. J Dev Behav Pediatr. 2006;27(6):459-69.

26. Cooper H. Cochlear implants: a practical guide. London: Whurr Publishers; 1991.

27. - Wiley S, Meinzen-Derr J, Grether S, Choo DI, Hughes ML. Longitudinal functional performance among children with cochlear implants and disabilities: a prospective study using the Pediatric Evaluation of Disability Inventory. Int $\mathrm{J}$ Pediatr Otorhinolaryngol. 2012;76(5):693-697. Using the PEDI, this article describes measurable improvements in all 3 domains comparing pre-implant and 1-year post-implant children with $D D$.

28. Berrettini S, Forli F, Genovese E, et al. Cochlear implantation in deaf children with associated disabilities: challenges and outcomes. Int J Audiol. 2008;47(4):199-208.

29. - Palmieri M, Berrettini S, Forli F, et al. Evaluating Benefits of Cochlear Implantation in Deaf Children With Additional Disabilities. Ear Hear. 2012;33(6):721-730. Their results show that children with additional disabilities have substantial progress in all areas of the $D A D Q$.

30. Meinzen-Derr J, Wiley S, Creighton J, Choo D. Auditory skills checklist: clinical tool for monitoring functional auditory skill development in young children with cochlear implants. Ann Otol Rhinol Laryngol. 2007;116(11):812-8.

31. Wiley S, Meinzen-Derr J, Choo D. Auditory skills development among children with developmental delays and cochlear implants. Ann Otol Rhinol Laryngol. 2008;117(10):711-8.

32. - Meinzen-Derr J, Wiley S, Grether S, Choo DI. Language performance in children with cochlear implants and additional disabilities. Laryngoscope. 2010;120(2):405-413. An article reporting a small group of post-implant patients with $D D$ that improved on either expressive or receptive language skills over time using the NVCQ.

33. Edwards LC, Frost R, Witham F. Developmental delay and outcomes in paediatric cochlear implantation: implications for candidacy. Int J Pediatr Otorhinolaryngol. 2006;70(9):1593-600.

34. Holt RF, Kirk KI. Speech and language development in cognitively delayed children with cochlear implants. Ear Hear. 2005;26(2):132-48

35. Boons T, Brokx JP, Dhooge I, et al. Predictors of spoken language development following pediatric cochlear implantation. Ear Hear. 2012;33(5):617-39.

36. Geers AE, Moog JS. Evaluating the benefits of cochlear implants in an education setting. Am J Otol. 1991;12(Suppl):116-25.

37. • Nikolopoulos TP, Archbold SM, Wever CC, Lloyd H. Speech production in deaf implanted children with additional disabilities and comparison with age-equivalent implanted children without such disorders. Int J Pediatr otorhinolaryngol. 2008;72(12):18231828. Speech Intelligibility Rasting (SIR) scale was used to evaluate implanted children with additional disabilities and 
compared them with age-equivalent implanted children without such disorders in a follow up period of 5 years.

38. Gerard JM, Deggouj N, Hupin C, et al. Evolution of communication abilities after cochlear implantation in prelingually deaf children. Int J Pediatr Otorhinolaryngol. 2010;74(6):642-8.
39. Mugno D, Ruta L, D’Arrigo VG, Mazzone L. Impairment of quality of life in parents of children and adolescents with pervasive developmental disorder. Health Qual Life Outcomes. 2007;5:22. 